\title{
État de l'anthropologie du développement au Mali
}

\author{
Yaouaga Félix Koné
}

\section{CpenEdition}

Journals

Édition électronique

URL : http://journals.openedition.org/apad/352

DOI : 10.4000/apad.352

ISSN : 1950-6929

\section{Éditeur}

LIT Verlag

Édition imprimée

Date de publication : 15 décembre 1991

\section{Référence électronique}

Yaouaga Félix Koné, «État de l'anthropologie du développement au Mali », Bulletin de I'APAD [En ligne] 2 | 1991, mis en ligne le 28 juin 2006, consulté le 07 septembre 2020. URL : http:// journals.openedition.org/apad/352 ; DOI : https://doi.org/10.4000/apad.352

Ce document a été généré automatiquement le 7 septembre 2020.

Bulletin de I'APAD 


\title{
État de l'anthropologie du développement au Mali
}

\author{
Yaouaga Félix Koné
}

1 Anthropologie, développement : une science et un concept qui, pour nous, Africains au sud du Sahara, sont indissociables des divers contacts que l'Afrique a vécu avec d'autres continents, d'autres civilisations. L'une s'est donnée pour mission la compréhension des pratiques sociales locales, l'autre s'acharne à la transformation des réalités économiques des pays étiquetés "en voie de développement".

2 A l'origine des contacts se trouve vraisemblablement un désir de développement, quand bien même celui-ci est tourné incontestablement vers la satisfaction des besoins du conquérant. En effet, les colonies devront être une garantie pour l'avenir et l'on entrevoyait la possibilité d'y dégager des excédents qui serviraient à développer la "mère patrie" (cf C. Rondeau 1980). Cette orientation du sens du développement a fortement influencé l'anthropologie (incluant tous les chercheurs en sciences sociales, plus particulièrement les sociologues, historiens et ethnologues) dans le choix de ses objets et déterminé la nature et l'importance de la demande dont elle a été l'objet à travers les différentes périodes de nos histoires économiques.

3 A défaut d'une anthropologie de la conquête, il y a eu implicitement une anthropologie de l'administration (qualitativement différente de l'anthropologie politique) ou au service de celle-ci. Cette anthropologie pour l'administration se découvre à travers les différents rapports des administrateurs. Une administration au service des grandes maisons de commerce dont les premières activités renvoient à la fameuse notion "d'économie de pillage". Ce développement de soi par le pillage de l'autre ne ressentait pas le besoin de l'éclairage d'une quelconque science sociale spécialisée. Les acteurs de ce développement se sont donc contentés d'un "savoir ethnologique naïf". Un savoir bâti sur des préjugés idéologiques négatifs, niant à l'autre les valeurs fondamentales de l'Homo Sapiens Sapiens, lointains échos de cette affirmation de Kant: "les nègres d'Afrique n'ont reçu de la nature que le goût des sornettes" (Observations sur le sentiment du beau et du sublime, 1764). Cette sociologie naïve a débouché sur la conception d'un mode de développement par la contrainte. Une recette largement 
utilisée dès l'aube de la colonisation et pendant longtemps, quand bien même elle se fit plus subtile. Elle généra les prélèvements directs, le travail forcé etc... Paresseux par essence, le nègre ne travaille que contraint, il faut donc l'obliger à créer des richesses (cf L. Tauxier). Dans cette optique, il fut signifié à l'indigène l'obligation de cultiver du coton, de l'arachide et de faire des plantations. A ce stade, le colonisateur jouait déjà le second acte de son développement : celui de la "mise en valeur" des colonies initiée à partir des années 1920.

4 Au delà du lourd tribut payé, les colonies ont tout de même hérité de certaines infrastructures. Au Mali, l'Office du Niger est une illustration de cette nouvelle orientation du développement. Cependant, là aussi, il a manqué les études préalables du social. Par conséquent, la réalité sociale n'a pas été prise en compte. Cette opération avait son consultant en sciences sociales en la personne de D. Zahan et pourtant n'a prévalu qu'une seule logique, celle du pouvoir conquérant, mue par l'unique préoccupation de la rentabilité économique. La dimension humaine dans une optique humanitaire faisait scandaleusement défaut.

5 En somme, ce qui a caractérisé les actions de développement des colonies est la mise à l'écart de la contribution des sciences sociales. Cette attitude n'est pourtant pas significative d'une absence totale de l'anthropologie. Elle était présente et même jouissait des structures et de l'assistance de l'administration du développement. Cependant, celle-ci n'a pas daigné faire appel aux compétences de celle-là, alors que le Mali a fait l'objet de l'intérêt d'ethnologues de très grande renommée. Sont de ceux-là L. Tauxier, C. Monteil, M. Delafosse, M. Griaule, G. Dieterlen, D. Zahan. Les trois premiers se sont investis à circonscrire géographiquement et à comprendre les caractéristiques physiques et mentales des ethnies. Les derniers se sont intéressés davantage au système de croyance des Dogon et des Bambara. Les uns et les autres ont produit un savoir sur des réalités typiquement intemporelles, savoir "coupé des vrais problèmes sociaux" cruciaux d'une Afrique en proie à des mutations accélérées. Est-ce là l'explication de l'indifférence qui a longtemps marqué les rapports développement/ sciences sociales? On est tenté de le penser. En effet, à présent encore, leur collaboration reste relativement timide. La nécessité d'une indispensable coopération, émise maintes fois après les échecs en série des Opérations de Développement au lendemain des indépendances, n'est pas encore unanimement acceptée et mise en application par tous les développeurs. Pourtant l'exigence de la compréhension des représentations et des logiques sociales, à un moment ou un autre du développement, est formellement affinée dans les études de faisabilité des projets. Il est ainsi demandé aux sciences sociales "un renouvellement des objets". A cette fin, certains départements ministériels se sont dotés de services en sciences sociales. Les créations de l'I.S.H (Institut des Sciences Humaines), de l'I.E.R. (Institut d'Économie Rurale), de la S.N.E.D. (Société Nationale d'Étude pour le Développement) relèvent de cette exigence. D'autres instituts et cabinets d'études, de statuts divers tels que l'A.M.R.A.D. (Association Malienne de la Recherche Action pour le Développement), I.M.R.A.D. (Institut Malien de La Recherche Appliquée au Développement), le G.R.A.D. (Groupe de Recherche Action pour le Développement) ont vu le jour. Toutes ces institutions. quel que soit leur statut (étatique ou privé). peuvent être consultées. Elles sont toutes plus ou moins tournées vers la recherche appliquée au développement.

6 Cette offre de savoirs anthropologiques est incontestablement significative d'une demande effective. Progressivement les sciences sociales sont impliquées dans le 
développement. Toutefois, cette implication reste marginale et restreinte. D'une manière générale, elle se situe soit au début de l'Opération de Développement. pour sa mise en œuvre, soit à la fin, pour l'évaluation. Exceptionnellement l'anthropologue est associé à la conception, à l'exécution et à l'appréciation d'une action de développement. Le chercheur en sciences sociales a le sentiment de n'être sollicité que pour cautionner ou diagnostiquer et/ou faire l'autopsie des Opérations. Son intervention se situe le plus souvent en aval. Pourtant, anthropologues et développeurs gagneraient à conjuguer dans la durée leurs efforts. L'éclairage constant et rationnel des premiers donnerait aux seconds davantage de possibilités de réussite, et alors seulement leur regard diachronique sur la réalité sociale serait le gage d'une approche parfaite du changement social.

\section{BIBLIOGRAPHIE}

Amsene J. L., 1990, Logiques Métisses, Paris, Payot.

Boirai P., Lanteri J.F., Olivier de Sardan J.P.(sous la direction de), 1985, Paysans, experts et chercheurs en Afrique noire, Paris, CIFACE - Karthala.

Le Bris E., Le Roy E. et Leimdorfer F. (études réunies et présentées par), 1982, Enjeux fonciers en Afrique noire, Paris, Karthala.

Rondeaux C., 1980, La société senoufo du sud Mali (1870-1950) De la tradition à la dépendance, Thèse de doctorat de $3 \mathrm{e}$ cycle, Paris VIT.

\section{AUTEUR}

\section{YAOUAGA FÉLIX KONÉ}

Institut des Sciences Humaines, Bamako. 